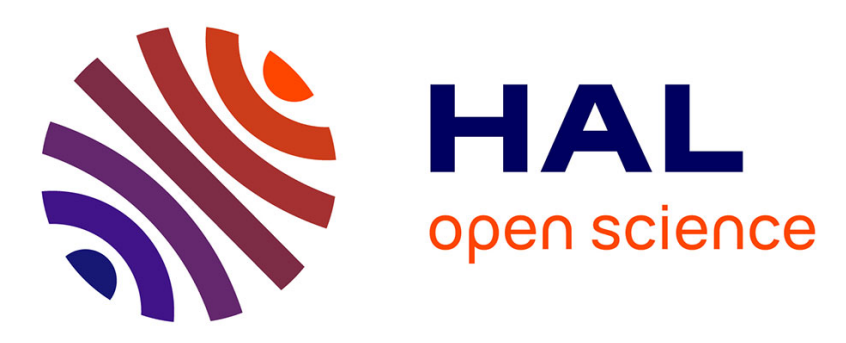

\title{
High-Q Optical Resonators for Stabilization of High Spectral Purity Microwave Oscillators
}

Pierre-Henri Merrer, Aude Bouchier, Houda Brahimi, Olivier Llopis, Gilles Cibiel

\section{- To cite this version:}

Pierre-Henri Merrer, Aude Bouchier, Houda Brahimi, Olivier Llopis, Gilles Cibiel. High-Q Optical Resonators for Stabilization of High Spectral Purity Microwave Oscillators. European Frequency and Time Forum - International Frequency Control Symposium (EFTF-IFCS 2009), Apr 2009, Besançon, France. 4 p. hal-00383911

\section{HAL Id: hal-00383911 https://hal.science/hal-00383911}

Submitted on 13 May 2009

HAL is a multi-disciplinary open access archive for the deposit and dissemination of scientific research documents, whether they are published or not. The documents may come from teaching and research institutions in France or abroad, or from public or private research centers.
L'archive ouverte pluridisciplinaire HAL, est destinée au dépôt et à la diffusion de documents scientifiques de niveau recherche, publiés ou non, émanant des établissements d'enseignement et de recherche français ou étrangers, des laboratoires publics ou privés. 


\section{High-Q Optical Resonators for Stabilization of High Spectral Purity Microwave Oscillators}

\author{
P.-H. Merrer, A. Bouchier, H. Brahimi, O. Llopis \\ LAAS-CNRS \\ Université de Toulouse ; UPS, INSA, INP, ISAE ; LAAS \\ Toulouse, France \\ bouchier@laas.fr
}

\author{
G. Cibiel \\ CNES \\ Toulouse, France
}

\begin{abstract}
Different high-Q optical resonators are presented in this communication. Our aim is to use them to develop compact high performances microwave oscillators. The optical quality factor of a quartz disk used as a whispering gallery modes resonator has been measured to be higher than $10^{9}$. We also investigate the potentialities of high- $Q$ fibre ring resonators. The performances of an optoelectronic oscillator at $10 \mathrm{GHz}$ based on such a passive fibre ring resonator are presented here.
\end{abstract}

\section{INTRODUCTION}

In order to design compact and high performances microwave oscillators, new resonators are investigated. But the dimensions of microwave resonators are intrinsically large, especially for cm-long wavelengths, and their quality factors also decrease when the frequency increases. Actually, the whispering gallery modes sapphire resonators present the best performances around $10 \mathrm{GHz}$, with an unloaded quality factor close to $10^{5}$.

An interesting method to reduce resonators dimensions is to carry the microwave on another wave, like acoustic or optical waves for example. In this study, we have chosen to use an optical carrier, here a $1.5 \mu \mathrm{m}$-laser. Thus microwave resonators become optical ones, with lower dimensions due to shorter wavelengths. It is thus possible to design devices that are more compact than the actual microwave ones. In this case, the optical resonator creates an optical frequency comb with microwave spacing. To each mode of this optical comb corresponds an optical $\mathrm{Q}$ factor $\left(\mathrm{Q}_{\mathrm{opt}}\right)$ and an equivalent $\mathrm{RF} \mathrm{Q}$ factor $\left(\mathrm{Q}_{\mathrm{RF}}\right) . \mathrm{Q}_{\mathrm{RF}}$ is the product of $\mathrm{Q}_{\mathrm{opt}}$ and the $\mathrm{RF}$ to optical frequencies ratio (1).

$$
Q_{R F}=Q_{o p t} \frac{f_{R F}}{f_{o p t}}
$$

Due to this relation, in opposition to the case of microwave resonators, the RF Q factor increases with the RF frequency. Nevertheless the high frequency ratio between microwave (around 10 to $20 \mathrm{GHz}$ ) and optics (about $194 \mathrm{THz}$ ) constrains the optical quality factor to be higher than $10^{9}$ at $1.55 \mu \mathrm{m}$ to guaranty a RF quality factor at $20 \mathrm{GHz}$ higher than $10^{5}$. In this case, these optical resonators can allow better performances than microwave components.

In the microwave photonics domain, optical resonators are an alternative method to the classical optical delay lines, which are easy to use and actually well known. These lines are few-km-long optical fibres, and their RF quality factors can reach a value of $10^{6}$ at $10 \mathrm{GHz}$. They have been used to stabilize oscillators [1] but are bulky devices and there thermal stabilization is difficult.

An interesting alternative to these optical delay lines is proposed by the mini optical resonators [2,3]. These devices are spheres, torus or disks, in which the optical wave circulates on the whispering gallery modes. These structures have to be transparent at $1.55 \mu \mathrm{m}$, and are constituted from silica, quartz, calcium $\left(\mathrm{CaF}_{2}\right)$ or magnesium fluoride $\left(\mathrm{MgF}_{2}\right)$ and so on... As presented here, these resonators have really high optical quality factors (from $10^{8}$ to $10^{10}$ at $1.55 \mu \mathrm{m}$ ), but their use is delicate. A last approach is to use the ability of fibered optics, which has been developed for telecommunications around $1.55 \mu \mathrm{m}$, and we present here the principle and use of fibre ring resonators [4]. We also present an optoelectronic oscillator (OEO) based on one of these passive fibre ring resonators.

\section{STUDY OF DIFFERENT MiCROWAVE PHOTONICS RESONATORS}

\section{A. The Whispering Gallery Modes Resonators}

We have studied two types of whispering gallery modes (WGM) resonators presenting a low volume: a silica sphere with a diameter of $3.3 \mathrm{~mm}$ and a quartz disk, with a diameter of $7.7 \mathrm{~mm}$. The whispering gallery modes are excited with the laser at $1.55 \mu \mathrm{m}$, which carries the microwave. The laser used for the characterization of these resonators is an amplified erbium-doped fibre laser from Koheras. It presents a really low linewidth of around $1 \mathrm{kHz}$ for a delay of $120 \mu \mathrm{s}$. It is tunable on a frequency range of $120 \mathrm{GHz}$ thanks to the variation of its temperature, with a precise tunability on a 
range of $2 \mathrm{GHz}$ with a piezoelectric component. This pigtailed laser is launched in the WGM resonators thanks to the evanescent waves coming from a tapered or a half-tapered fibre. This fibre presents a waist of around $2 \mu \mathrm{m}$ on a length of a few millimetres (Fig. 1), and is fabricated by Laseo (France) [5]. Due to the tunability of the laser, we have measured the optical quality factor of the silica sphere and the quartz disk with a method of scan. This consists in a scan of the frequency of the laser to explore the different optical modes of the frequency comb due to the resonator. We can thus measure the distance between two modes (the free spectral range or FSR), and the full-width at half-maximum of a mode. The linewidth of the laser is really low and is smaller than the width of a resonant mode. We can thus have a good resolution of the main resonant modes.

For the fused silica sphere, we have determined an optical quality factor of about $210^{8}$ at $1.55 \mu \mathrm{m}$ with a strong decrease to $10^{6}$ after two years. This drop of the optical quality factor is due to the degradation of the resonator surface quality, probably caused by chemical reaction with the atmospheric water. We are thus mainly dealing with the second WGM resonator in the follow of this communication.
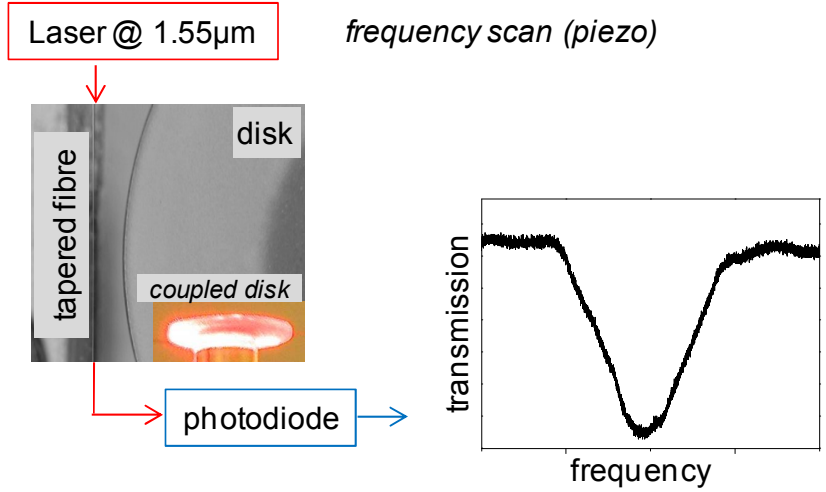

Figure 1. Schematical setup of the characterization of the whispering gallery modes resonators with the scan method.

In the case of the quartz disk, with a diameter of $7.7 \mathrm{~mm}$, we have measured a free spectral range of $8 \mathrm{GHz}$ and a width of the main resonant modes of $47 \mathrm{kHz}$ (Fig. 1). With these measurements, we can determine an optical quality factor for this resonator of $410^{9}$ at $1.55 \mu \mathrm{m}(\sim 194 \mathrm{THz})$. This really high optical quality factor let us expect RF quality factors around $410^{5}$ at $20 \mathrm{GHz}$. Nevertheless these compact resonators present some disadvantages. Firstly, the coupling of the laser in the resonator with tapered fibres and the excitation and selection of whispering gallery modes is difficult and can be unstable. Secondly, these optical resonators are highly transverse multimode and numerous gallery modes can be excited in the resonator. This is clearly visible with a scan of the frequency of the single-frequency low linewidth laser. The stabilization of the laser frequency on a frequency resonance is thus really difficult. We are studying alternative coupling techniques to improve the coupling efficiency and stability, like the use of prisms [6] or angle-polished fibres instead of the tapered fibres. Thus, although we still study these compact WGM resonators, which are really promising in terms of high optical quality factors, investigations on different types of resonator are also interesting.

\section{B. The Fibre Ring Resonators}

The optical fibres are particularly adapted for utilization at telecommunication wavelengths. We can thus use transverse single-mode fibres at $1.55 \mu \mathrm{m}$ like the classical SMF28 fibre. A resonator can be built with such fibres, leading to a transverse single frequency comb. On another side, laser coupling in the resonator become really easy if we use pigtailed fibre lasers. In this case, the coupling is stable, and the system can be integrated in a compact device. We have thus studied a resonator based on the fibre ring described in [4].This ring is fabricated with two single-mode fibres (Corning SMF28) linked with two low-loss fibered couplers (typically with a ratio of $99 / 1 \%$ ) as described on Fig. 2 . The connection between fibres inside the ring is made by splicing the fibres. The total length of the ring is from 1 to $20 \mathrm{~m}$, leading to a free spectral range comprised between 10 and $200 \mathrm{MHz}$.

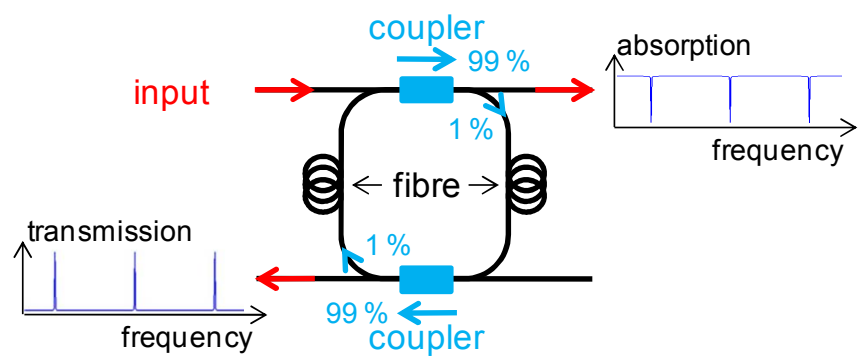

Figure 2. Scheme of the fibre ring resonator and resonant modes in transmission and absorption.

The simulation of such a fibre ring is explained in [4]. The optical couplers are described with their coupling coefficients and losses. The model takes also into account the losses of the fibre splices (about $0.02 \mathrm{~dB}$ ). The fibered couplers used for the rings are experimentally characterized. Optical quality factors from $210^{8}$ for a ring length of $1 \mathrm{~m}$ to $510^{9}$ for a $20 \mathrm{~m}$-long ring can be expected according to the simulation. We have thus studied experimentally a ring with a total length of $20 \mathrm{~m}$.

The scan method used for the study of the whispering gallery modes resonators allowed us to check a free spectral range for this ring of $10 \mathrm{MHz}$. We have also observed that these resonators are really sensitive to some external influences due to their high quality factor. Thermal effects are the most difficult to control. They are mainly due to temperature variations of fibre environment and also to the laser launching inside the fibre ring. This effectively modifies the local temperature in the fibre, especially in the coupler, and leads to local changes of index and length. For the couplers, a change in temperature can also lead to a modification of coupling characteristics and to the appearance of losses. Globally, these thermal variations shift the resonance frequency of these resonators. It is thus necessary to stabilize the laser on a resonance frequency of the ring, as shown on the experimental setup of the Fig. 3.

The laser stabilization is obtained thanks to a PoundDrever-Hall (PDH) loop $[7,8]$. The phase modulation is 
realized with a lithium niobate modulator. The error signal from this loop is sent on a proportional differential integrator. The correction is applied on the piezoelectric component of the Koheras laser. The laser frequency is thus precisely tuned.

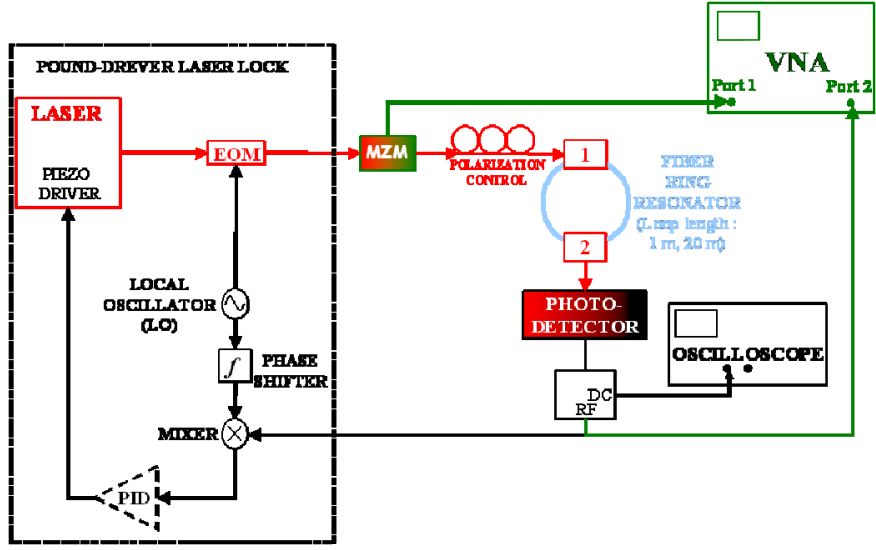

Figure 3. Setup for the test of microwave filtering by the optical resonator.

When the laser is stabilized on the resonator, it is possible to modulate it with microwaves. In this aim, we use a MachZehnder Modulator (MZM). Lateral modes are thus explored by scanning the modulator frequency, thanks to a microwave network analyzer (Fig. 3). The resonator spectrum is then stable and we can measure a full-width at half-maximum of $65 \mathrm{kHz}$ (Fig. 4).

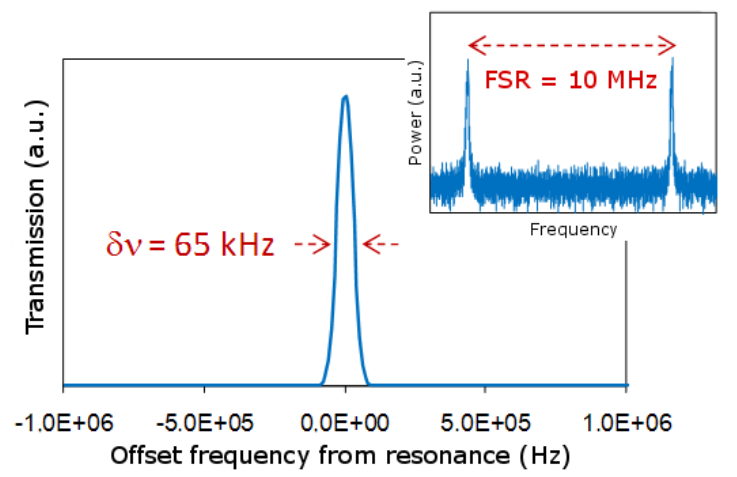

Figure 4. Resonance of the stabilized 20m-long fibre ring and free spectral range in inset.

The optical quality factor determined with this method is comparable to the simulation, with a value of $310^{9}$ for a $20 \mathrm{~m}$ long fibre ring resonator composed with $99 / 1 \%$ fibered couplers. In the microwave range, this performance leads to an equivalent RF quality factor of $310^{5}$ at $20 \mathrm{GHz}$, which is largely higher than what can be obtained directly with microwave resonators.

Nevertheless some difficulties have been observed when the oscillator has been built with this resonator: an important phase noise component has been measured. A new fibre ring resonator, which solves this problem, has thus been developed (patent pending). But it presents a lower optical quality factor, around $210^{8}$ at $1.55 \mu \mathrm{m}$. Despite its low quality factor, a first oscillator has been assembled with this fibre ring resonator.

\section{DEMONSTRATION OF AN OPTOELECTRONIC OSCILLATOR BASED ON AN OPTICAL RESONATOR}

From the setup on the Fig. 3, we have realized an optoelectronic oscillator (OEO) with a frequency of $10 \mathrm{GHz}$. The principle of the experimental setup is depicted on Fig. 5.

The output signal from the photodiode is amplified. It contains the different frequency beats between the resonant modes of the fibre ring resonator.

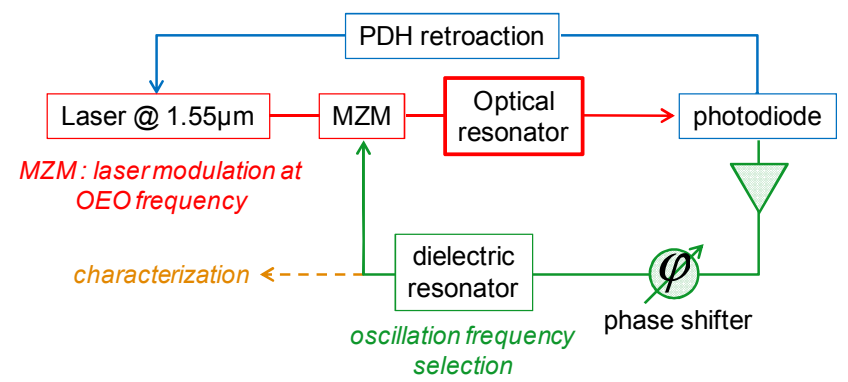

Figure 5. Principle of the optoelectronic oscillator at $10 \mathrm{GHz}$.

In order to build an oscillator around $10 \mathrm{GHz}$, we have used a dielectric resonator that filters a frequency range of about $3 \mathrm{MHz}$ around $10 \mathrm{GHz}$ in order to select only one mode from the frequency comb of the optical resonator (Fig. 6).

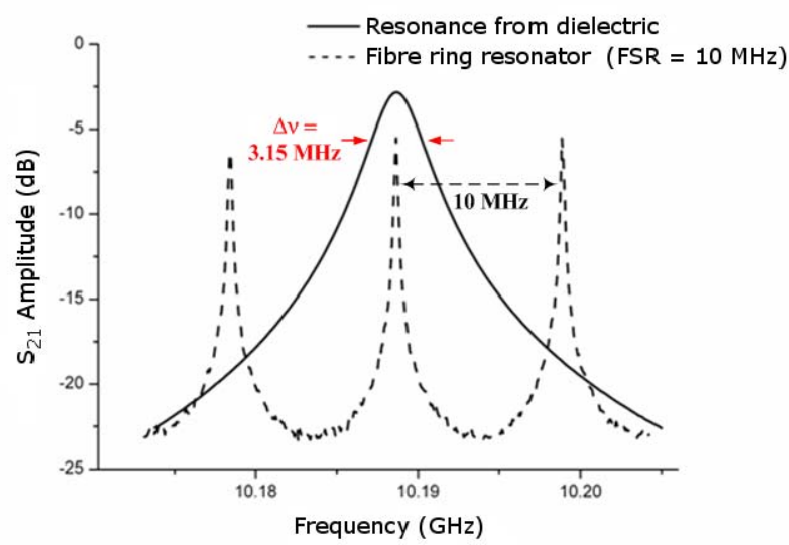

Figure 6. Comparison around $10 \mathrm{GHz}$ of the transfert function of the dielectric resonator and the free spectral range of the $20 \mathrm{~m}$-long fibre ring.

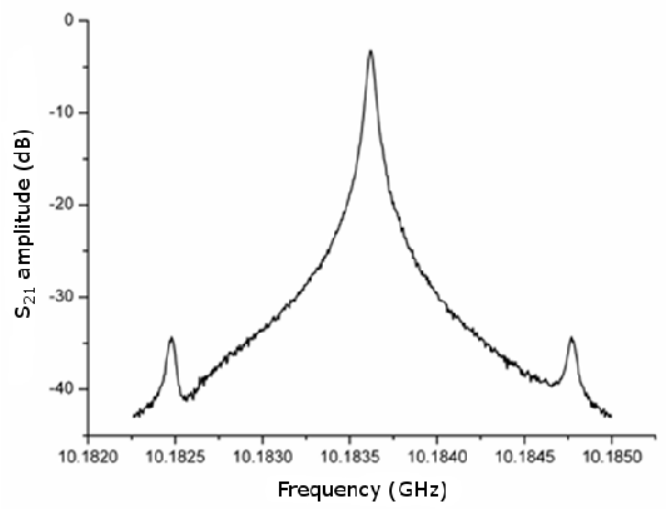

Figure 7. Resonance around $10 \mathrm{GHz}$ of the fibre ring resonator after filtering with the dielectric resonator. 
Thus, the RF oscillation starts on this mode only (Fig. 7). It is stable and no other spurious modes are observed.

The spectrum of this oscillator and its phase noise spectrum have been measured thanks to an Agilent E5052 signal analyzer. The results are presented on Fig. 8.

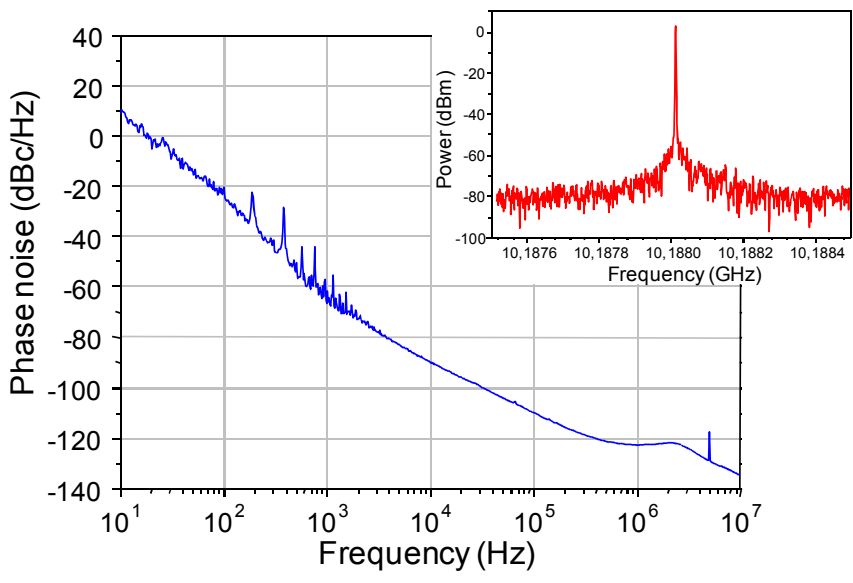

Figure 8. Phase noise measurement and spectrum (in inset) for the optoelectronic oscillator at $10 \mathrm{GHz}$.

The phase noise spectrum presents different slopes. Between $10 \mathrm{~Hz}$ and $1 \mathrm{kHz}$, the phase noise is dominated by the $1 / \mathrm{f}$ frequency noise, which is characterized by a slope of $-30 \mathrm{~dB} / \mathrm{dec}$. The spurious peaks that are visible between 0.1 and $1 \mathrm{kHz}$ can be due to thermal or mechanical fluctuations of the fibre ring resonator. The white frequency noise is characterized by a slope of $-20 \mathrm{~dB} / \mathrm{dec}$ from 1 to $400 \mathrm{kHz}$ and is related to the signal to noise ratio in the RF oscillating loop. The phase noise floor appears after $400 \mathrm{kHz}$, and has a value of about $-120 \mathrm{dBc} / \mathrm{Hz}$. The final decrease of the noise is linked to the filtering by the dielectric resonator.

The frequency $f_{c}$ at the frontier between white frequency noise and the phase noise floor is related to the loaded microwave quality factor $\left(\mathrm{Q}_{\mathrm{RF}}\right)$ by the relation (2)

$$
f_{c}=\frac{f_{R F}}{2 \cdot Q_{R F}}
$$

with $f_{R F}$ the microwave frequency [9]. With a corner frequency of $0.4 \mathrm{MHz}$, the loaded microwave quality factor is $1.210^{4}$ at $10 \mathrm{GHz}$, which is in good agreement with an optical quality factor of $210^{8}$ at $1.55 \mu \mathrm{m}$ (eq. 1 ).

In spite of its good quality factor, this oscillator presents a relatively high phase noise level. This phenomenon is mainly due to the transmission losses in the optical device. These losses are added to the low coupling rate between the resonant ring and the transmission output of the resonator (Fig. 2), and contribute to decrease the signal to noise ratio at the output of the resonator, and thus to increase the phase noise of the oscillator. A second factor is linked to the different $1 / \mathrm{f}$ phase noise sources in the optical components that have not been optimized for this first demonstration. We are actually working to improve these parameters in our future oscillators.

\section{CONCLUSION}

Optical resonators allow thus to achieve really high quality factor in the microwave range, while they guarantee low dimensions. Whispering gallery modes resonators are potentially interesting for really compact oscillators, if solutions for an efficient assembling can be found to propose integrated (or semi-integrated) coupling for these devices. Fibered resonators like fibre ring resonators are easier to integrate and use. The optoelectronic oscillator presented in this communication is based on such a resonator and is, up to our knowledge, the first one of this type to be published. Its performances in terms of phase noise are still modest and close to the ones of a dielectric resonator oscillator (DRO). Nevertheless, its potentialities in terms of microwave quality factors are really promising and the current development of a new optimized resonator will allow us to highly improve the performances of this oscillator.

\section{REFERENCES}

[1] X.S. Yao and L. Maleki, "Progress in the optoelectronic oscillator - a ten year anniversary review," 2004 IEEE Microwave Theory and Tech. Symp. Digest, pp. 287-290, 2004.

[2] M.L. Gorodetsky, A.A. Savchenkov and S.V. Ilchenko, "Ultimate Q of optical microsphere resonator," Opt. Letters, vol.21 (7), pp. 453-5, 1996.

[3] D.K. Armani, T.J. Kippenberg, S.M. Spillane and K.J. Vahala, "Ultrahigh-Q toroid microcavity on a chip," Nature, vol.421, pp. 925-8, 2003.

[4] P.H. Merrer, O. Llopis and G. Cibiel, "Laser stabilization on a fiber ring resonator and application to RF filtering," IEEE PTL, vol.20 (16), pp. 1399-1401, 2008

[5] P.H. Merrer, O. Llopis, S. Bonnefont, P. Feron and G. Cibiel, "Microwave filtering using high Q optical resonators," Proc. of the European Microwave Conf., Amsterdam 2008.

[6] V.S. Ilchenko, J. Byrd, A.A. Savchenkov, A.B. Matsko, D. Seidel and L. Maleki, "Miniature oscillators based on optical whispering gallery mode resonators," Frequency Control Symposium, 2008 IEEE International , pp.305-308, 19-21 May 2008

[7] R. V. Pound, "Electronic frequency stabilization of microwave oscillators," Rev. Sci. Instrum., vol. 17 (11), pp. 490-505, 1946.

[8] R.W.P. Drever, J.L. Hall, F.V. Kowalski, J. Hough, G.M. Ford, A.J. Munley and H.Ward, "Laser phase and frequency stabilization using an optical-resonator,“ Appl. Phys. B., vol. 31, no. 2, pp. 97-105, Jun. 1983.

[9] D.B. Leeson, "A simple model of feedback oscillator noise spectrum," Proc. of the IEEE, vol. 54, no. 2, pp. 329-330, Fev. 1966. 